\title{
La chicha, primera cancioneta popular del Perú republicano
}

La chicha, the first popular canzonetta of republican Peru

\author{
Lucas Borja-Rojas ${ }^{1}$
}

\section{Resumen}

La chicha fue una cancioneta compuesta por José Bernardo Alzedo y José de la Torre Ugarte, los mismos autores de nuestro Himno Nacional, que fue cantada por el pueblo en aquellos días en que se declaró la Independencia del Perú, hace doscientos años. El autor la rescata de su olvido presentándonos la letra y partitura musical.

Palabras claves: cancioneta, música, popular, Independencia del Perú.

\section{Abstract}

The chicha was a canzonetta composed by José Bernardo Alzedo and José de la Torre Ugarte, the same authors of our National Anthem, which was sung by the people in those days when the Independence of Peru was declared, two hundred years ago. The author rescues it from oblivion by presenting us with the lyrics and musical score.

Keywords: canzonetta, music, popular, Independence of Peru.

La chicha, título de la tradicional canzoneta de José Bernardo Alzedo y José de la Torre Ugarte que hemos olvidado, al igual que parece ocurriría con la chicha de jora, que cada día tomamos menos, pese a sus extraordinarias cualidades.

Pocos tienen presente que aquel día, 28 de Julio de 1821, después que San Martín proclamara la Independencia del Perú, en la hoy Plaza de Armas de Lima, Plazuela de la Merced, la de Santa Ana y

\footnotetext{
${ }^{1}$ Compositor, intérprete y recopilador.
}

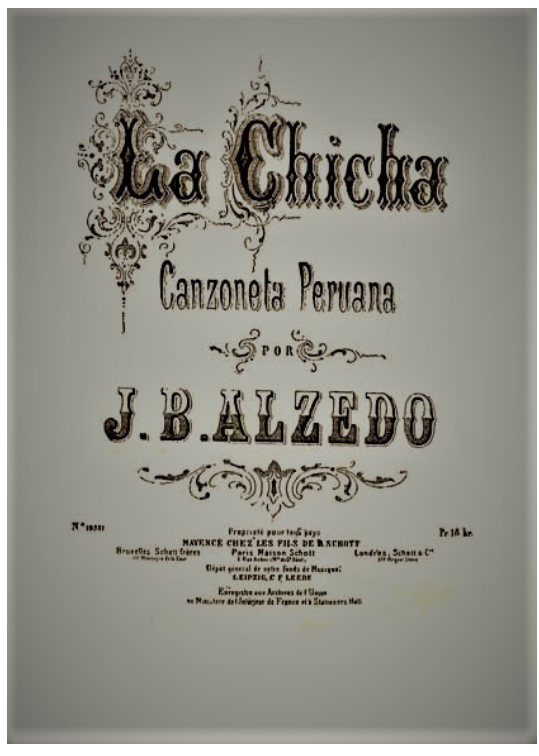

en la del Congreso, se reunieron los manifestantes, y precedidos por el Libertador, marcharon al Teatro Principal (hoy Teatro Segura); y allí, con gran regocijo se entonó en repetidas oportunidades, la canzoneta, que lleva por título La chicha, compuesta por los mismos autores del Himno Nacional, Alzedo y De la Torre Ugarte, ad portas de nuestra Independencia, evocando nuestra chicha de jora, licor ancestral, de nobles virtudes, así como otros potajes nacionales, incluyendo además bellos mensajes a la nueva nación independiente y que gracias a una copia de la partitura de la época escrita por los propios autores, hemos podido saborear, no bien llegó a nuestras manos, hace varios lustros. 
CORO

\begin{abstract}
¡PATRIOTAS EL MATE DE CHICHA LLENAD
Y ALEGRES BRINDEMOS POR LA LIBERTAD

Y ALEGRES BRINDEMOS POR LA LIBERTAD! (BIS)
\end{abstract}

CUBRA NUESTRAS MESAS EL CHUPE Y QUESILLO YEL AJI AMARILLO, EL CELESTE AJI

Y A NUESTRAS CABEZAS LA CHICHA SE VUELE

LA QUE HACERSE SUELE DE MAIZ O MANI

LA QUE HACERSE SUELE DE MAIZ O MANII (BIS)

PATRIOTAS EL MATE...

III

EL INCA LA USABA EN SU REGIA MESA CON QUE AHORA NO EMPIEZA QUE ES INMEMORIAL BIEN PUEDE EL QUE ACABA, PEDIR SE RENUEVE EL POTO EN QUE BEBE O SU CAPORAL.

EL POTO EN QUE BEBE O SU CAPORAL (BIS)

PATRIOTAS EL MATE...

v

OH LICOR PRECIOSO, TU LICOR PERUANO LICOR SOBREHUMANO, MITIGA MI SED. OH NECTAR SABROSO DEL COLOR DEL ORO

DEL INDIO TESORO ;PATRIOTAS BEBED! DEL INDIO TESORO ¡PATRIOTAS BEBED! (BIS)

PATRIOTAS EL MATE...

VII

AL CÁLIZ AMARGO DE TANTOS DISGUSTOS, SUCEDAN LOS GUSTOS, SUCEDA EL PLACER. DE NUESTRO LETARGO A UNA DESPERTEMOS Y TAMBIEEN LOGRAMOS, LIBRES POR FIN SER. Y TAMBIEN LOGRAMOS, LIBRES POR FIN SER.

PATRIOTAS EL MATE...
II

ESTA ES MÁS SABROSA, QUE EL VINO Y LA SIDRA QUE NOS TRAJO LA HIDRA, PARA ENVENENAR.

ES MUY ESPUMOSA Y YO LA PREFIERO A CUANTO EL IBERO PUDO CODICIAR. A CUANTO EL IBERO PUDO CODICIAR.

PATRIOTAS EL MATE...

\section{IV}

EL SEVICHE VENGA, LA GUATIA ENSEGUIDA QUE TAMBIÉN CONVIDA Y EXITA A BEBER. TODO INDIO SOSTENGA, CON EL POTO EN MANO QUEA TODO TIRANO HA DE ABORRECER. QUE A TODO TIRANO HA DE ABORRECER. (BIS)

PATRIOTAS EL MATE...

VI

SOBRE LA JALEA DEL AJI UNTADA

CON MANO ENLAZADA EL POTO APURAD.

YESTE BRINDIS SEA EL SIGNO QUE DAMOS

A LOS QUE ENGENDRAMOS EN LA LIBERTAD. A LOS QUE ENGENDRAMOS EN LA LIBERTAD.

PATRIOTAS EL MATE...

VIII

GLORIA ETERNA DEMOS AL HÉROE DIVINO QUE NUESTRO DESTINO, CAMBIADO HA, POR FIN SU NOMBRE GRABEMOS, EN EL TRONCO BRUTO DEL ARBOL QUE EL FRUTO, DEBE A SAN MARTIN. DEL ARBOL QUE EL FRUTO, DEBE A SAN MARTIN. (BIS)

PATRIOTAS EL MATE...

LETRA FIEL AL DOCUMENTO ORIGINAL DE DON JOSÉ DE LA TORRE UGARTE.

RECOPILACIONES LUCAS BORJA ROJAS

Acta Herediana vol. 64, N², julio 2021 - diciembre 2021 


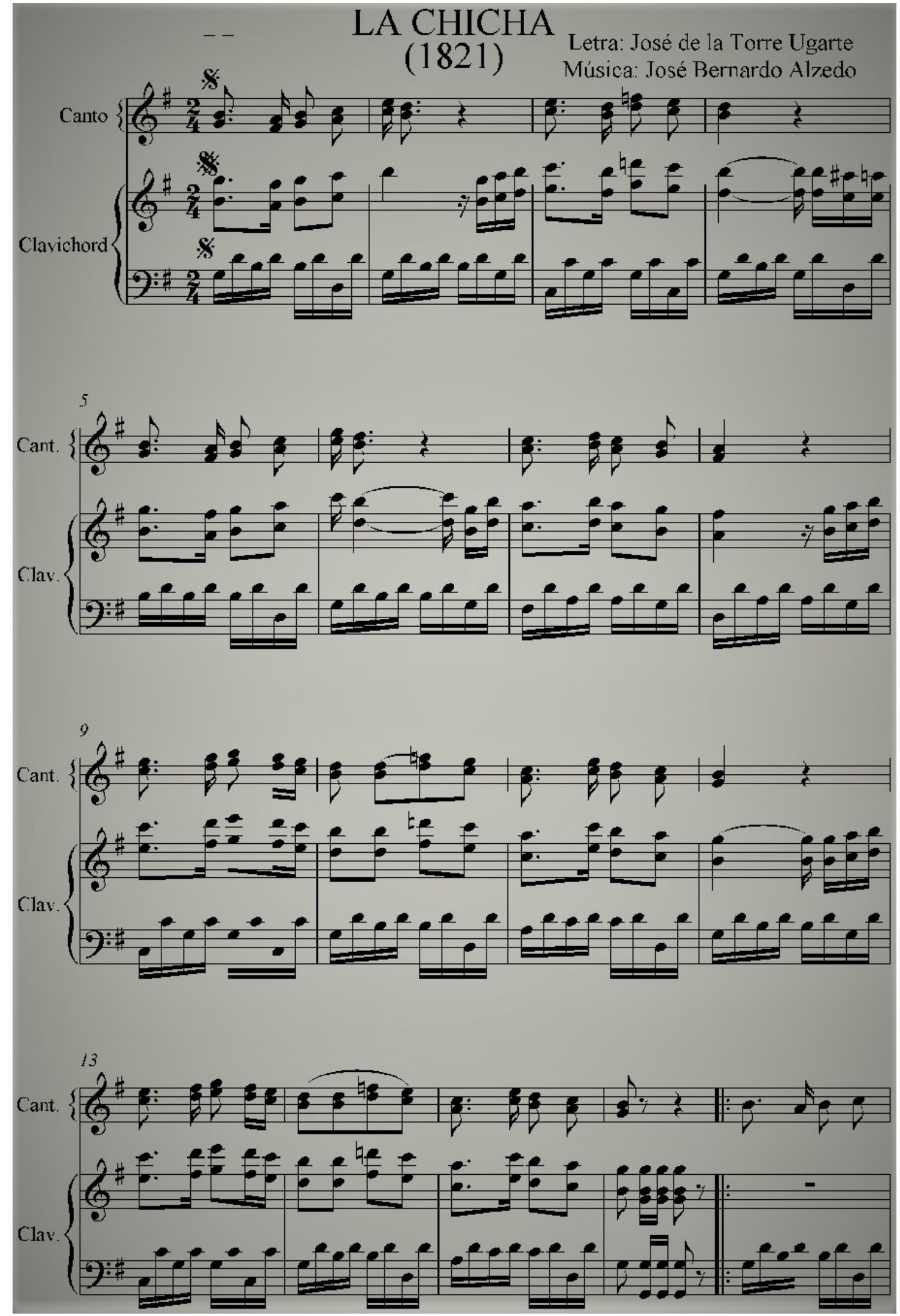

Acta Herediana vol. 64, N², julio 2021 - diciembre 2021 


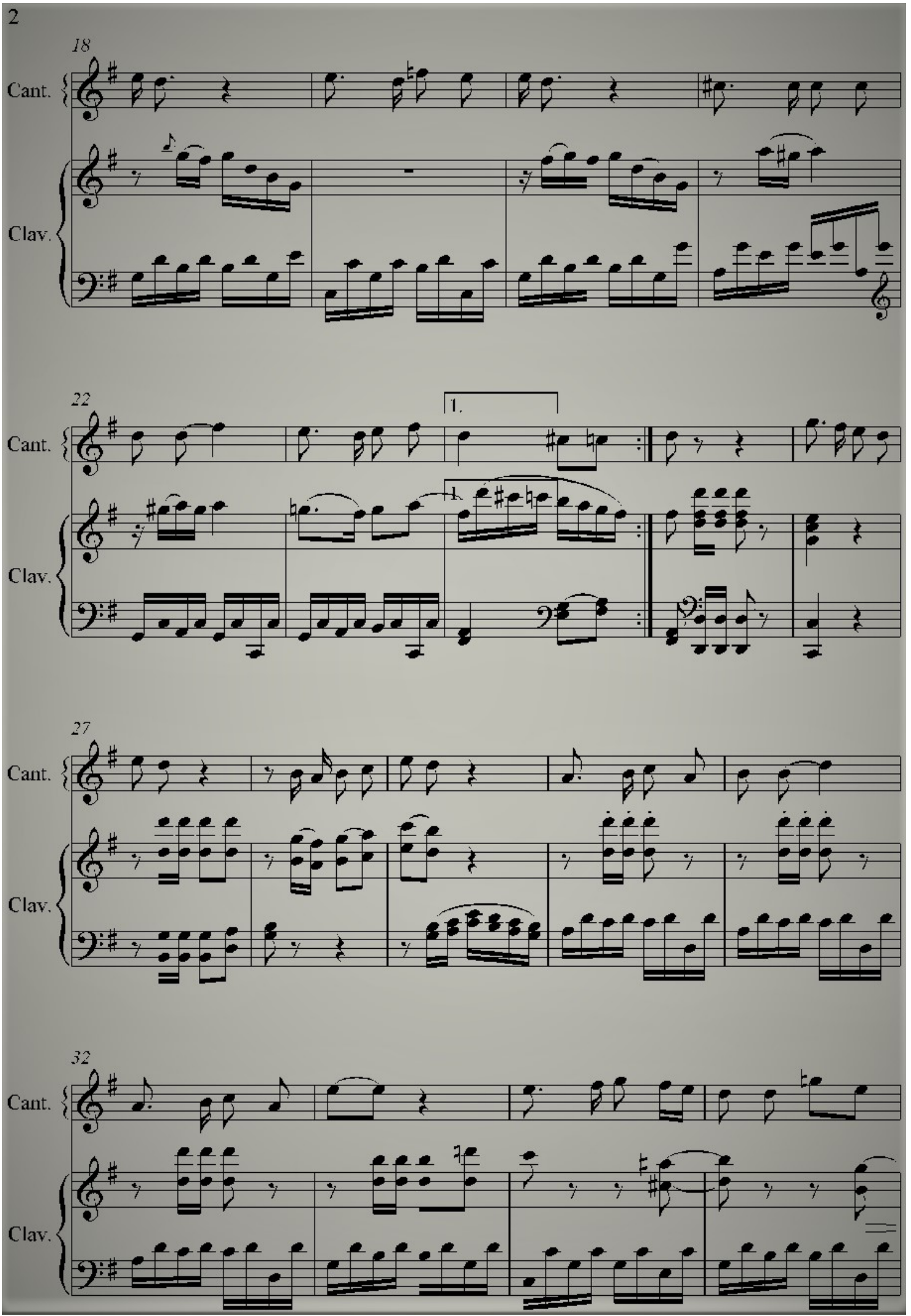

Acta Herediana vol. 64, N², julio 2021 - diciembre 2021 


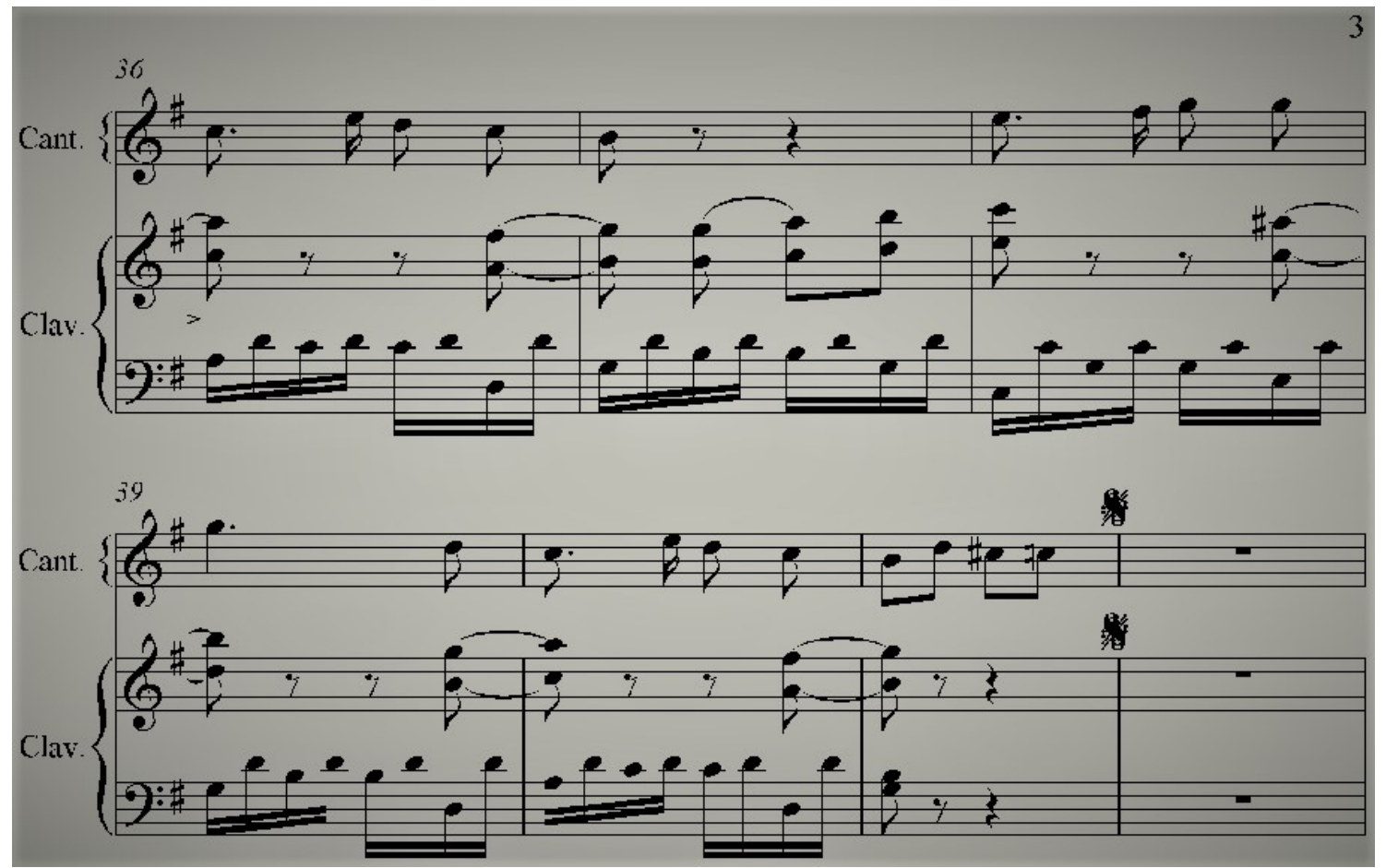

En el año 2004, exactamente el miércoles 21 de Julio, los que concurrimos al Auditorio del Centro Cultural Ricardo Palma de Miraflores, pudimos compartir una maravillosa experiencia que nos transportó a aquel 28 de Julio de 1821, al escuchar en interpretación del Trío Patria, con la pista de la versión original tomada de dicha partitura $\mathrm{y}$ que llevada al sistema computarizado, por nuestra cancionista Luisa Ramos, integrante de aquel trío, quien además por la bondad del sistema mencionado consigue aplicar el instrumento que se utilizó en aquella magna fecha, es decir el clavicordio, bajo cuyo audio de acompañamiento entonaron la alegre canción a tres voces, evocando la versión que escucharan los que acompañaran al libertador San Martín el día de la Juramentación de nuestra Independencia.

Hoy hace 200 años, según refieren los escritos de la época, en ese entonces el público deliraba de alegría y con mucho fervor patriótico seguía, acompañando con las palmas al antiguo clavicordio que armonizaba los versos de La Chicha, como repito, de los mismos autores que como se sabe, también compusieron la marcha, hoy Himno Nacional del Perú.

Con ocho estrofas, que precede un coro o estribillo, a través de los versos de esta canzoneta podemos apreciar cómo se resalta lo auténticamente nacional o pertenecientes a nuestro ancestro prehispánico, a lo incaico enfrentando a lo impuesto de fuera; lógicamente, en contra de la dominación española. la armonía que le infiere Alzado es maravillosa.

En consecuencia, es de suma trascendencia histórica esta canción, la primera que lanza al viento, el sentimiento popular en la fecha libertaria. No era para menos, pues el mensaje de la canzoneta de marras es extraordinario, nos exhorta a brindar 
por la libertad con nuestra ancestral chicha de jora. "¡Patriotas el mate de chicha llenad, y juntos brindemos por la libertad! Es así mismo enjundioso cuando entona:

"Cubra nuestras mesas el chupe y quesillo... Esta es más sabrosa, que el vino y la sidra, que nos trajo la hidra, para envenenar...El inca la usaba en su regia mesa...El seviche venga, la guatia enseguida... Oh licor precioso, tu licor peruano...Licor sobrehumano, mitiga mi sed... Oh néctar sabroso del color del oro...Del indio tesoro ipatriotas bebed!...

Y, para terminar:

Gloria eterna demos al héroe divino

Que nuestro destino, cambiado ha, por fin.

Su nombre grabemos, en el tronco bruto

Del árbol que el fruto, debe a San Martin
La vieja canción, hoy yace olvidada, como olvidada nuestra incomparable y legendaria chicha de jora. En Lima es recordada tan sólo en asociaciones de provincias y en reducidos círculos de patriotas que al escucharla brindan como impulsados por las altisonantes arengas de hogaño que mueve a peruanidad: ¡Patriotas el mate de chicha llenad, y alegres brindemos por la libertad! ¡Viva el Perú!

Lima, julio del 2021

\section{Correspondencia:}

Luisa Borja

luisabrm18@gmail.com

Fecha de recepción: 29-07-2021.

Fecha de aceptación: 20-08-2021.

Conflicto de interés: ninguno, según el autor.

Financiamiento: por el autor. 